(di) https://doi.org/10.37843/rted.v9i2.135

\title{
Conflicto Escolar en la Educación Rural del Nororiente de Colombia
}

\section{School Conflict in Rural Education in Northeast of Colombia}

\author{
Alveiro Álvarez-Ovallos ${ }^{1}$, Aurora Gélvez-López ${ }^{2}$ y Jemay Mosquera-Téllez ${ }^{3}$
}

\section{อ}

\section{EDICIÓN: 曲:-CIVTAC}

Recibido: 12/mayo/2020

Aceptado: 10/junio/2020

Publicado: 25/septiembre/2020

Colombia

${ }^{2}$ Colombia

${ }^{3}$ Colombia

\section{IIIIInstitución}

${ }^{1}$ Universidad de Pamplona

${ }^{2}$ Universidad de Pamplona

${ }^{3}$ Universidad de Pamplona

\section{Correo Eletrónico}

1alveiro403@gmail.com

2aurora.gelvez@hotmail.com

3jemay.mosquera@unipamplona.edu.co

\section{(1) ORCID}

${ }^{1}$ https://orcid.org/0000-0003-4023-7136 ${ }^{2}$ https://orcid.org/0000-0002-5168-580X ${ }^{3} \mathrm{https}$ //orcid.org/0000-0001-5989-5644

\section{Citar así: C4.APA / IEEE}

Álvarez-Ovallos, A., Gélvez-López, A. \& Mosquera-Téllez, J. (2020). Conflicto Escolar en la Educación Rural del Nororiente de Colombia. Revista Tecnológica-Educativa Docentes 2.0, 9(2), 5-15.

https://doi.org/10.37843/rted.v9i2.135

A. Álvarez-Ovallos, A. Gélvez-López y J. Mosquera-Téllez, "Conflicto Escolar en la Educación Rural del Nororiente de Colombia", RTED, vol. 9, n. ${ }^{\circ}$ 2, pp. 5-15, sep. 2020.

\section{Resumen}

El conflicto escolar, problemática que atañe a toda comunidad educativa, tiene repercusiones que suscitan preocupación por cuanto sus manifestaciones afectan física, emocional y académicamente a los implicados. Sin embargo, el conflicto puede ser tomado como oportunidad de progreso personal para mejorar la convivencia y optimizar procesos institucionales; por lo tanto, el objetivo del trabajo se centró en caracterizar tipos de conflicto presentes en diferentes espacios escolares y sus formas de resolución en la Institución Educativa Edmundo Velásquez, ubicada en zona rural del departamento Norte de Santander, Colombia. La investigación fue de enfoque cualitativo, descriptivo e interpretativo con corte transversal, la población de estudio está conformada por 36 estudiantes del grado sexto de la básica secundaria y el instrumento de obtención de información es la observación participante, la cual permitió registrar en un diario de campo eventos, comportamientos que alteran el clima escolar. Los resultados demuestran prevalencia de conductas disruptivas en estudiantes varones, intolerancia y ausencia de valores, mientras que las conclusiones evidencian necesidad de mejorar relaciones interpersonales, promover armonía, generar cambios estructurales de carácter sociocultural, regular la resolución de conflictos a través de políticas educativas y mitigar situaciones con incidencia negativa en todo el estudiantado.

Palabras clave: Convivencia escolar, agresión, violencia, conductas disruptivas.

\section{Abstract}

The school conflict, a problem that affects every educational community, has repercussions that raise concern because its manifestations affect those involved physically, emotionally and academically. However, the conflict can be taken as an opportunity for personal progress to improve coexistence and optimize institutional processes; Therefore, the objective of the work was focused on characterizing the types of conflict present in different school spaces and their forms of resolution in the Edmundo Velásquez Educational Institution, located in a rural area of the Norte de Santander department, Colombia. The research was of a qualitative, descriptive and interpretive approach with a cross-section, the study population is made up of 36 students of the sixth grade of secondary school and the instrument for obtaining information is the participant observation, which allowed recording in a diary of field events, behaviors that alter the school climate. The results show the prevalence of disruptive behaviors in male students, intolerance and the absence of values, while the conclusions show the need to improve interpersonal relationships, promote harmony, generate structural changes of a socio-cultural nature, regulate conflict resolution through educational policies and mitigate situations with negative impact on the entire student body.

Keywords: School conflict, school coexistence, disruptive behaviors, aggression. 


\section{Introducción}

La población rural de Colombia es parte fundamental en el desarrollo socioeconómico del país; sin embargo, en el contexto de la región nororiental del país, dicha ha sufrido permanentemente múltiples situaciones adversas, representadas en abandono estatal, corrupción política y un conflicto armado presente desde hace varias décadas con diferentes grados de intensidad en diversas zonas del país. El nororiente colombiano se ha caracterizado por ser un territorio de constante alteración del orden público, específicamente el departamento Norte de Santander, cuya población rural asciende alrededor del $22 \%$ de la población total, según datos demográficos emitidos por la gobernación del departamento.

Aspectos relacionados con la vulnerabilidad multidimensional presente en contextos rurales colombianos, han sido expresados históricamente a través de un gran número de particularidades trasmitidas de generación en generación y expresadas en bajas garantías de superación económica, social o cultural. En lo educativo, se evidencia un gran porcentaje de analfabetismo de población adulta, como condición arraigada en costumbres y valores transmitidos por sus progenitores, siguiendo un instinto natural; de tal forma que la población rural se encuentra apegada "a sus costumbres y valores, poco acepta ayuda externa por temor a perderlos, además, esta población vive unas alejadas de las otras" (Salazar, 2010, p. 2). Además, el poco contacto social entre poblaciones rurales dispersas, aunado a una idiosincrasia campesina de carácter conservador, se revierte en apatía y renuencia al contacto con personas ajenas a la comunidad, desde una posición de desconfianza o recelo.

Ahora bien, como factor primordial para cerrar brechas de desigualdad social entre población rural y urbana, es sin lugar a duda la educación, como derecho fundamental consagrado en la Constitución Política Colombiana (artículo 67), lo que puede contribuir de forma significativa en la transformación de niños o adolescentes de la zona rural. Lo anterior, sin desconocer los grandes desafíos característico en estas zonas de difícil acceso, pero con enormes posibilidades de originar el aprendizaje del conflicto desde una condición situacional ubicada en el centro de las relaciones sociales, entendida como pilar fundamental del desarrollo emocional, personal $\mathrm{o}$ académico de cada uno de los educandos (Cabrales,
Contreras, González \& Rodríguez, 2017, p. 6).

Al enfatizar en la educación rural se evidencian primeramente diferentes situaciones por las que atraviesan cada uno de los estudiantes en sus vivencias o desafíos propios cada contexto (De Sousa, 2009), ya sean de carácter familiar, grupal o comunitario, social o económico. Seguidamente, se manifiesta una baja cobertura educativa inherente a sectores rurales con poca inversión en infraestructura, escasos recursos económicos, ausencia de material didáctico y baja conectividad. Lo anterior, constata la ineficiente aplicabilidad de políticas educativas por parte del Gobierno nacional, departamental o municipal para este sector.

Actualmente, la población joven rural se encuentra en busca de mejores oportunidades con el fin de contribuir al desarrollo regional; por lo tanto, es necesario implementar políticas educativas pertinentes, enfocadas no solamente se forme a niños y adolescentes en aspectos académicos, sino también se brinde apoyo psicosocial enfocado en desarrollar el potencial innato en cada uno de ellos, desde un proyecto de vida orientado al mejoramiento de condiciones básicas del ser humano desde lo individual con impacto comunitario. Por lo tanto, se debe fomentar desde temprana edad la importancia la Educación como camino para lograr reconstruir el tejido social.

Por otro lado, los procesos educativos, bien sean de carácter urbano o rural, presentan una problemática social recurrente en diversas entidades e instituciones que buscan prestar este servicio. En término de convivencia escolar, este es indudablemente un aspecto de gran importancia en las relaciones humanas, razón por lo cual se debe defender fehacientemente, sin dejar a un lado los impactos que provoca una baja relación entre pares, como por ejemplo desacuerdos, comunicación inadecuada, etcétera, condiciones generadoras de conflictos interpersonales o alteraciones del ambiente escolar, de diferentes altercados cuya solución habitualmente no se da de una forma adecuada, en tanto se presentan casos asociados al uso de fuerza como medio de respuesta (Caballero, 2010, p. 155).

De esta manera, un trato personal desequilibrado va desquebrajando relaciones establecidas a lo largo del tiempo dentro de un grupo o comunidad estudiantil; imposibilita una aplicación efectiva de mecanismos de resolución de conflictos, 
independientemente del grado de agresión; crea fisuras cada vez más grandes e irreconciliables; fracturando lazos de amistad forjados previamente. Lo anterior, es ampliamente demostrado dentro de escenarios escolares, donde educandos se rehúsan a acoger asertivamente formas de solución de altercados o se dejan conducir por emociones momentáneas, como condición previa de afectaciones de tipo verbal, psicológico o físico, con implicación en su propio bienestar aunado a repercusiones en personas cercanas (Mina \& Rangel, 2012, p. 13).

Por el contrario, una muy buena relación interpersonal permite asimilar diferencias existentes en otras personas, en cuanto a opiniones, ideas o percepciones, respetar diversos modos de actuación y ser solidario ante situaciones de adversidad, todo ello mediante escenarios de cooperación en equipo para cumplir metas comunes o individuales.

\section{Convivencia Escolar}

Convivencia escolar corresponde a una acción de aprender a vivir juntos con tolerancia, de convivencia entre diferentes personas que hacen parte del contexto escolar, de manera armónica y respetuosa frente a la violencia, agresión e indisciplina; así como un conjunto de relaciones y elementos necesarios para lograr propósitos o resultados de aprendizaje forjadores de procesos educativos integrales (García \& Ferreira, 2005).

Por ello, es conveniente propender por ideales imprescindibles en toda institución educativa asociados a interpretar adecuadamente cualquier manifestación de diversidad, asegurando en sus educandos parámetros de óptimos de convivencia, en la medida que "el respeto, la tolerancia y las buenas acciones deben imperar en cada uno, a pesar de todas las situaciones que como ser humano podemos estar viviendo" (Arredondo, 2015, p. 54).

Sin lugar a duda, desde hace mucho tiempo, escuelas e instituciones educativas se conciben, cada vez con más fuerza como espacios aptos para empezar a compartir múltiples situaciones interpersonales, bien sea positivas o negativas; para formar individuos en todos sus ámbitos, integrándolos en una comunidad de iguales, enseñándoles a convivir idealmente bajo un buen clima (Bahamón, Pacheco \& Sáenz, 2015, p. 6).

Por lo tanto, convivencia escolar corresponde a una noción, cuyo encauce de tipo formativo, es considerado como fundamento esencial de procesos de formación ciudadana, como un factor clave de formación integral de estudiantes; en ese sentido, un componente importante está relacionado con la misión educativa de enseñar a estudiantes prestos a captar conocimiento, mejorar comportamiento, descubrir sus habilidades y demostrar valores, como prerrequisitos para vivir en paz y armonía con el prójimo; a este respecto, se puede inferir lo importante que resulta desarrollar procesos de gestión formativa para asegurar, de manera efectiva, una convivencia escolar tendiente a prevenir o atenuar situaciones de violencia escolar (Sandoval, 2014, p. 160).

De igual manera, es primordial propiciar un excelente ambiente orientado a consolidar relaciones sociales favorables, como elemento valioso para salvaguardar identidades culturales locales, especialmente asociado a escenarios educativos ruarles conducentes al desarrollo del aprendizaje. Por ello, es de suma importancia ofrecer a nuestros educandos un ambiente social placentero, donde haya una armonía sin igual entre los niños, tanto a nivel escolar como familiar, todo esto para asegurar resultados con impacto positivo en la sociedad (López, Carvajal, Soto \& Urrea, 2013, p. 386).

Para algunos investigadores como Cajiao (2015) y Patiño (2014), muchos escenarios generadores de conflictos en cuanto a convivencia en aulas de clase se deben a diversos factores propios del entorno docente; específicamente, hacen referencia al uso inadecuado de herramientas pedagógicas, generalmente usadas para desarrollar cualquier actividad curricular, sin tener en cuenta aspectos particulares del proceso formativo. Por otra parte, se considera indispensable contar con estudiantes motivados, como condición para lograr un aprendizaje significativo, mientras que el uso de metodologías inadecuadas o herramientas didácticas inapropiadas impiden lograr dicho objetivo, generando estudiantes disruptivos, producto de docentes con bajo dominio de grupo complementado con un manejo inadecuado de altercados en el aula.

Entre otros factores se evidencia también poco trabajo colaborativo o en equipo dentro de las instituciones educativas, así como escasez de liderazgo pedagógico en docentes, sumado a baja estima manifestada en frecuentes enemistades entre directivos, maestros, estudiantes, padres de familia. 
Actualmente, se advierte una recurrente producción de situaciones conflictivas al interior de las instituciones educativas, como condición generadora de problemas de convivencia, de situaciones que se convierten en motivo alarma para la comunidad académica y su entorno familiar, situación que permea a toda la comunidad.

Por lo tanto, situaciones asociadas a convivencia escolar se convierten cada vez más en una preocupación sentida de maestros y directivos en instituciones educativas rurales. Obviamente, no se puede desconocer la influencia de situaciones interpersonales manifestadas a lo largo del tiempo, cuyos involucrados pueden presentar características antagónicas o interrelaciones positivas, de forma que la convivencia es un reto ineludible en el marco del cual se deben tomar procedimientos significativos por parte de las instituciones educativas, orientados a configurar una óptima convivencia escolar (Abenza, 2015).

\section{Conflicto Escolar}

Cuando se aborda el conflicto, es necesario asumir su manifestación en situaciones normales, presentes en toda sociedad, en algunas ocasiones producto de un hecho social consustancial a la vida en comunidad. Sin embargo, las discrepancias en el ser humano son una constante histórica, hecho fácil de constatar a través de diferentes sucesos o aspectos cotidianos registrados en cualquier época, sin importar estrato socioeconómico, raza, edad, religión, o condiciones espacio temporales. Incluso, cuando ocurren variaciones sociales en la vida de cualquier ser humanos, estas podrían ser una consecuencia para imputar de modo mayoritario, aun cuando no de manera absoluta, al conflicto (Silva, 2008).

Históricamente, el conflicto ha sido tildado o asociado principalmente a una situación nefasta, donde los sucesos negativos se pudieron en gran medida evitar. Por cierto, siempre ha sido relacionado con situaciones de violencia en sus diferentes manifestaciones, así como a la guerra en el ámbito internacional.

Actualmente, el conflicto es considerado como un proceso natural, característico en cualquier dinámica asociada a relaciones humanas presentes en el entorno, como un aspecto clave del desarrollo individual. En ese sentido, las formas de enfrentar un conflicto dependen directamente de las percepciones relacionadas con su manejo, es decir, con aspectos asociados a su significado social (González \& Rojas, 2014, p. 14).

Independiente de su tipo o características, el conflicto puede alcanzar caminos destructivos, además de producir una constante reiteración de los hechos en los cuales subsisten relaciones incompatibles u hostiles; sin embargo, también se puede obtener aspectos funcionalmente positivos dependiendo de los hechos, de quienes están implicados o de su nivel social, emocional o psicológico (Domínguez \& García, 2003).

A su vez, el conflicto hace ver la incapacidad de mantener la cordura e impide tener aplomo para controlar situaciones cotidianas. De igual forma, conduce a sufrir algún tipo de pérdida, separación o dependencia no deseada, induce a manifestar aptitudes, desarrollar habilidades positivas $\mathrm{o}$ negativas supuestamente inexistentes. El conflicto promueve situaciones inimaginables que rondan en límites desconocidos, perturba la tranquilidad, interrumpe nuestros sueños, sacude nuestras vidas, nos saca del nido, nos priva de la comodidad, nos obliga a valernos por nosotros mismos, alejándonos de todas aquellas personas que dan sentido a la vida (De Souza, 2009, p. 8).

Entonces, cada vez adquieren mayor importancia los diversos acontecimientos cercanos al conflicto, los desacuerdos ocasionados por distintas circunstancias se convierten en puntos neurálgicos, ya sean de carácter nacional o internacional, social o personal, se van acrecentando constantemente por motivos de índole ideológico, sexual, religioso, político o económico, llegando a suscitar una serie de respuestas que pueden originar acciones relacionadas con un aumento significativo del problema.

Los conflictos se originan sin distinción de contextos, por ejemplo, en la vida cotidiana, toda persona se enfrenta a circunstancias, en las cuales el tomar una decisión lo puede llevar a producir algún tipo de conflicto, sin embargo, esa decisión se puede convertir en oportunidades de cambio, o por el contario causa dificultades constantes.

Por tanto, el abordaje del conflicto es más complejo de lo que se cree, no es suficiente con nombrarlo o saber que existe, sino asumirlo también como un asunto de incumbencia general, sin excepción, sobre todo en la escuela, lugar donde se ha incrementado vertiginosamente, aunque, en este caso es conveniente diferenciarlo de manifestaciones 
relacionadas con actos violentos, pues todos los conflictos no son violentos (Aguilar \& Ariza, 2015, p. 33).

Por lo anterior, el entorno escolar es considerado como un sitio de socialización, de conocimiento, donde acuden niños o jóvenes a complementar su formación como sujetos pensantes, a apropiarse de entendimiento para el desarrollo de habilidades necesarias para una mejor comprensión de la realidad. También es un escenario donde se adquieren valores, hábitos, para su formación como sujetos democráticos. Es así como la escuela es llamada a dar respuesta a un sinnúmero de interrogantes emergentes de los procesos de interacción de niños o jóvenes en relación con dinámicas culturales, políticas, económicas, sociales, como condición que posibilita su actuación y conformación de sujeto dentro de una sociedad democrática en la cual expresa sus comportamientos habituales.

De igual forma, son reconocidos como problemáticas coligadas a conflictos dados en instituciones educativas, aquellos altercados representados en situaciones de incompatibilidad entre dos o más instancias. Sin embargo, existen dos tipos de conflicto entre alumnos: los resultantes de actitudes o situaciones personales y los originados en su interacción social, entre ellos o con los demás integrantes de la comunidad estudiantil. Los conflictos personales pueden caracterizarse como ansiedad, conducta para llamar la atención, inestabilidad emocional, exceso de impulso competitivo, autoconcepto desfavorable, negativismo, hiperactividad, aislamiento, desobediencia habitual, problemas motivacionales (Bustos, 2009, p.2). Los conflictos interpersonales pueden ser la conducta interruptora, agresión física, conducta verbal, conocimiento deficiente de las relaciones causa-efecto, conducta pasivo sugestionable, inmadurez social, relaciones interpersonales ineficientes (Ortiz, 2014, p. 68).

Algunos elementos generadores de conflictos entre estudiantes pueden ser provocados, tanto por las relaciones interpersonales de dominio, como por factores individuales de autoestima o egocentrismo; para otras personas, las causantes de los conflictos a menudo se «psicologizan», reduciéndose a diferentes tipos de necesidades humanas, un problema de interpretación subjetiva, de percepciones, es decir, asumiendo un origen de situaciones de conflicto en el entorno escolar, ligado a cuatro categorías: ideológico-científicas, de poder sociopolítico, estructurales y eminentemente humanas bien sea personales o interpersonales (Pérez \& Gutiérrez. 2016, p. 169).

Por su parte, una problemática relacionada con el conflicto presente dentro y fuera de establecimientos educativos corresponde a la agresividad, entendida como un componente del comportamiento antisocial, considerado como fenómeno complejo, interconectado con múltiples factores que contribuyen a la presentación de diferentes manifestaciones por parte de los estudiantes. El comportamiento agresivo suele presentarse en distintos lugares, tales como hogar, escuela o comunidad, con manifestaciones productoras de deterioro clínicamente significativo de actividades sociales, académicas o laborales cotidianas (Samper, Tur, Mestre \& Cortés, 2008, p. 432).

Algunos comportamientos frecuentes en esta línea están asociados a romper objetos de otras personas, destruir mobiliario de lugares públicos, agredir a otros sujetos, falsificar calificaciones del colegio, faltar a clase o llegar tarde intencionalmente, beber en exceso, consumir sustancias psicoactivas, "colarse" cuando hay que esperar un turno, ensuciar calles rompiendo botellas y arrojando basura, entre otros aspectos (Contini, Cohen, Coronel \& Mejail, 2012, p. 19). De este modo, diferentes manifestaciones agresivas en adolescentes se han ido englobando en función de tres dimensiones básicas: a) naturaleza o modo de expresión (física/verbal); b) dirección (directa/indirecta), y c) función motivadora (activa/pasiva) (Andreu, Peña \& Penado, 2013, p.734).

De igual manera, en un ambiente escolar se presenta toda serie de escenarios incorporados a situaciones de conflicto, de baja tolerancia o irrespeto, recurrentes ofensas verbales o burlas grotescas, provocaciones humillantes, amenazas, robos o destrucción de objetos personales. Por tanto, se requiere una sana convivencia escolar, facilitadora de una construcción colectiva del tejido social, cuyas mejoras se apropien de manera responsable por parte de la comunidad educativa; una convivencia escolar enfocada positivamente hacia una búsqueda consciente de constantes manifestaciones de afecto, asertividad 
comunicativa, empatía y responsabilidad, teniendo como prioridad el mejoramiento de cualquier tipo de relación entre alumno - profesor; todo ello fundamentado sobre bases pedagógicas de aprendizaje tendientes a favorecer ambientes de aprendizaje participativos e incluyentes, como condición para construcción de identidad a pesar de posibles diferencias (Marrugo, Gutiérrez, Concepción \& Concepción, 2016, p. 75).

Por su parte, una elevada prevalencia de comportamientos agresivos originadores de consecuencias negativas se relacionan significativamente con a) precaria salud física articulada a consumo elevado de drogas legales e ilegales; b) bajos niveles de bienestar emocional producto del estrés percibido, con sintomatología depresiva o baja satisfacción vital; c) déficit de habilidades sociales relacionadas con una mayor probabilidad de rechazo por parte de compañeros, aunado a escaso apoyo parental o comunicación ofensiva con sus padres; y d) diversos problemas escolares, tales como apatía por aprender o bajo rendimiento académico (Torregrosa, Inglés \& García, 2011).

En el contexto específico de las comunidades rurales, se observa que, tanto al interior del aula, como fuera de ella, existen constantes conflictos de convivencia, los cuales no se gestionan debidamente. Se evidencia naturalización de escenarios de agresión articulada a un bajo avance tecnológico característico en la comunidad rural, como condición influente en comportamientos inadecuados de educandos, en adquisición de actitudes impropias para el entorno rural, tales como uso de dispositivos electrónicos usados como puerta de escape a un mundo de mayor libertad; por ello, un gran reto subyacente en acciones propias del profesorado radica en las posibilidades para brindar condiciones necesarias en la transformación de hábitos o límites culturales establecidos en el recinto escolar (Mendoza, Cervantes \& Pedroza, 2016, p. 3).

\section{Metodología}

El estudio es de tipo cualitativo, con relevancia específica en relaciones sociales, de carácter interpretativo con corte transversal orientado hacia una comprensión de las conductas conflictivas reiterativas entre estudiantes dentro de un establecimiento de educación media, Institución
Educativa Edmundo Velásquez, ubicado en Norte de Santander, Colombia.

Desde esta perspectiva, la aproximación hacia la dinámica del conflicto escolar se realiza partiendo del reconocimiento de una realidad presente en el entorno formativo, cuya comprensión es clave para generar procesos de gestión de conflictos. Al respecto, se considera conveniente indagar desde un descubrimiento interpretativo asociado a hechos conflictivos presentes en estudiantes sujetos de estudio, asumidos a su vez como un todo integral para lograr una apreciación adecuada dentro de su contexto singular con perspectivas valiosas para profundizar en dinámicas de conflicto escolares (Melo, Montaño, Mora \& Pavas, 2009, p. 88).

\section{Población}

La población objeto de estudio corresponde a 36 adolescentes del grado sexto (20 de sexo masculino, 16 de sexo femenino), cuyas edades oscilan entre 11-13 años, pertenecientes al nivel de educación básica secundaria y correspondiente a la totalidad de estudiantes de grado sexto.

\section{Técnica de Recolección de Información}

Como técnica de recolección de información se utiliza el instrumento observación participante, enfocado a una "descripción sistemática de eventos, comportamientos y artefactos en el escenario social elegido para ser estudiado" (Marshall \& Rossman, 1989, p. 35). Para cumplir con el objetivo principal del trabajo dirigido a caracterizar los tipos de conflicto emergentes entre estudiantes de grado sexto, se aplica un instrumento recolección de información definido por los autores para determinar la regularidad de situaciones conflictivas $\mathrm{o}$ faltas cometidas, tanto dentro como fuera del aula de clase, en diferentes momentos del proceso formativos: llegada del estudiante, durante algunas clases elegidas aleatoriamente por hora o día escolar, en descansos,

Quehacer estudiantil entorno a actividades académicas sin participación docente, comedor escolar, transporte escolar y salida de clases. Con esto se pretende conocer, una gran variedad de situaciones conflictivas entre estudiantes. 


\section{Procedimiento}

La investigación se realiza en las siguientes fases:

1. Etapa de Diseño: Se planifica el proceso investigativo definiendo población a estudiar, metodología, técnica de recolección de información, procedimiento para análisis de resultados.

2. Fase del trabajo de campo: Se realiza un primer acercamiento donde, a través de observación informal, se obtendrá una caracterización del contexto, con la respectiva población sujeto de estudio.

3. Observación: Se aplica el instrumento y se realiza, a manera de diario de campo, un registro escrito de las acciones o sucesos, faltas producidas, grado de conflictividad en diferentes situaciones escolares. El registro presenta datos a diligenciar durante el tiempo de observación, tales como fecha/hora, lugar, descripción del conflicto, participantes en el conflicto, percance o impacto.

\section{Resultados}

El proceso de evaluación visual sobre estudiantes pertenecientes a grado sexto ha permitido realizar un seguimiento de acciones que estos realizan en diferentes situaciones que alteran su comportamiento normal, especialmente en el aula de clase, aunque también se indaga su comportamiento general.

Las conductas más frecuentes se han clasificado de la siguiente forma:

1. En el aula de clase: $\mathrm{Se}$ observa constantemente conductas disruptivas como levantarse continuamente, pasearse por el salón provocando malestar en los compañeros, provocar ruidos con los pupitres, generar sonidos con la boca (silbidos, risas burlonas) para desconcentrar al grupo, hablar de temas no relacionados con el proceso académico (música, programas televisivos, canciones, eventos deportivos) o realizar comentarios fuera de contexto para incomodar a sus compañeros. Como manifestaciones generadoras de conductas indisciplinadas se contemplan las siguientes: tirarse bolas de papel a la cara incitando al otro a reaccionar de forma violenta como motivo para su amonestación, colocar apodos para hacer sentir más al otro, decir mentiras para inculpar a los demás, usar palabras descomedidas para insultar a otros compañeros, tomar sin consentimiento pertenencias de los compañeros, esconder objetos personales, botar la basura al piso, salir de clase sin autorización, empujar, retar a pelear, colocar zancadillas.

En clases de educación física, teniendo en cuenta su contexto lúdico, dinámico, de contacto físico, se presentan algunas situaciones donde se pasa rápidamente del juego a agresiones físicas, dado su condición para una manifestación directa de diferentes emociones ligadas a competencia entre educandos o al afloramiento de rivalidades; por lo tanto, algunas acciones relevantes detectadas corresponden a aislamiento de algunos integrantes del grupo producto de su escaza habilidad deportiva, supremacía del grupo más fuerte en competencia sobre el resto, presencia de burlas por alguna acción equívoca de juego, generación de sensación de superioridad frente da estudiantes más débiles físicamente, manejo de palabras ofensivas para desaprobar errores de juego. Además, en algunos casos, se evidenció agresiones físicas producto del calor del juego.

Por otro lado, se observaron reacciones de enfado, nerviosismo, susto, enrojecimiento del rostro. En pocos casos, se manifiesta llanto por parte de alguna estudiante cuyas vivencias asociadas a algún tipo de violencia se reflejan en el aula, situación asumida como conflicto psicológico en la medida de los cambios repentinos de emociones (González, 2017, p. 83).

Otros espacios objeto de técnica de observación participante corresponden a tiempo de descanso, donde se presentan las siguientes conductas:

1. Recreo o Tiempo de Descanso: En estos espacios se observa constantemente acciones de indisciplina como empujarse entre ellos, insultos fuertes, palabras soeces, choques, encontronazos, señas de insultos con las manos, echarse agua cuando se encuentran en los baños. Al respecto, se toman acciones 
correctivas o se realiza algún tipo de intervención por parte de docentes presentes cuando estos perciben faltas de respeto o actitudes de pelea relacionadas con posibles agresiones verbales o físicas; los docentes generan espacio para el ofrecimiento de disculpar por pate del agresor o entre los implicados, también se realiza un llamado de atención verbal a quien realiza el acto. En otras situaciones, los estudiantes son llevados a oficinas administrativas donde se realizan anotaciones en el libro de disciplina, dependiendo del grado de afectación ocasionado a sus compañeros.

2. Comedor Escolar: Otro espacio donde objeto de observación de comportamientos estudiantiles corresponde al restaurante o comedor escolares, donde a raíz del poco tiempo para comer los alimentos, se presente muy baja acción asociada a alteración de convivencia pacífica entre estudiantes. Las únicas situaciones presentadas son el abucheo por parte de los estudiantes cuando alguien se salta el turno de puesto, ciertas palabras de rechazo cuando no encuentran un sitio libre donde comer, momento en el cual se dirigen a sus compañeros con mofa para presionarlo a ceder su espacio. Las acciones anteriores no evidencian una intervención por parte de docentes encargados de controlar la disciplina en estos espacios.

3. Transporte Escolar: Se realizó un acompañamiento con el fin de observar el comportamiento, tanto antes como después de llegar a clase. Se encontró una conducta variada dependiendo del momento formativo, es decir, en horas tempranas se presenta calma, con baja presencia de manifestaciones de inconformismo frente al servicio de transporte; sin embargo, el fin de jornada escolar, conjuntamente con el regreso a sus hogares genera aumento o alteración de conducta, así como presencia de situaciones, tales como como colocar apodos, burlarse por algo sucedido anteriormente; al respecto, generalmente se hace una amonestación verbal por parte del coordinador acompañante del recorrido. Por otro lado, en algunas ocasiones se pudo observar conflictos donde se buscaba imponer decisiones con el fin de obtener alguna clase de poder dentro del aula de clase, para de esta forma obligar a disciplina o viceversa, lo anterior se relaciona con sumisión, coartación, manipulación del otro a través del sarcasmo, castigo, reto, etc. Tales estrategias de control no solo coartan ciertas manifestaciones de libre expresión del alumno, sino también obstaculizan su desarrollo psicosocial al ser puesto en posición de desventaja o ser sometido a los designios de otra persona. Lo anterior, se manifestó en algunas ocasiones, con implicados conscientes de operar bajo determinadas estrategias de enfrentamiento conflictivo, pero sin percatar su accionar negativo en la medida del agrado o aprobación de cierto grupo de estudiantes.

Cabe destacar una baja presencia de cualquier tipo de situaciones conflictivas en las dinámicas e interacciones femeninas, independiente del lugar de donde estas se encuentren, no se agreden de forma física, pocas lo hacen verbalmente con referencia a rumores o comentarios subidos de tono, por medo de insultos, a raíz de apariencia física, como también poniendo en contra otras estudiantes para lograr apoderarse de la situación. Al no revestir gravedad, estos conflictos son solucionados entre ellas o por medo de una intervención oportuna del docente.

De acuerdo con la información obtenida, en el marco al cumplimiento de los objetivos planteados, se observa presencia de diferentes tipos de conflicto escolar, los cuales pueden ser categorizados mayormente de tipo físico con alto grado de implicación en procesos de convivencia escolar. Por su lado, la implicación en menor grado de las manifestaciones de tipo verbal genera un bajo impacto sobre las dinámicas de convivencia escolar.

Los resultados obtenidos evidencian un alumnado del grado sexto copartícipe de conductas disruptivas perturbadoras frente a un desarrollo normal del proceso de enseñanza aprendizaje; además, se encuentra diferentes acciones interrelacionadas, vinculados a intolerancia (causa muy común), así como algunos casos abuso de confianza entre estudiantes, ausencia de práctica de valores, recurrencia de egoísmo e irrespeto, como aspectos generadores de situaciones negativas con 
repercusiones en su formación académica y personal.

Además, de la información obtenida, se puede inferir una convivencia percibida por los estudiantes como regular frente a la interacción con sus compañeros. Dicho aspecto se encuentra asociado a vivencias personales negativas en su proceso formativo, lo cual fue detectado en razón a actitudes demostradas no solo por estudiantes involucrados en la situación conflictiva, sino también por aquellos ajenos o no involucrados en situaciones de alteración de la convivencia escolar.

Por su parte, el grado de intervención de los docentes frente a ocurrencia de algún tipo de conflicto es variable; en algunos casos, intervienen pocas veces, en otros son muy activos dando soluciones en el momento de los sucesos. De igual manera, se evidenció el uso de sanciones disciplinarias a estudiantes con conductas hostiles o comportamientos de difícil manejo, manifestadas en rebajas de calificaciones tanto de alguna materia específica como en evaluaciones de tipo comportamental, llamados de atención verbal, anotaciones en libro de disciplina o llamados a padres de familia (acudientes) del educando. En ese sentido, se evidencia bajo manejo de estrategias disciplinarias para normalización de clases por parte de algunos docentes.

\section{Conclusiones}

Convivencia escolar, conflictos, agresiones, son temas cuya lenta pero permanente presencia conduce a su incorporación en el vocablo de todos aquellos que hacemos parte de ambientes educativos, situación también familiar para entornos formativos rurales, máxime si se tiene en cuenta situaciones de orden público, desplazamiento forzado $\mathrm{o}$ problemáticas de orden social, comunes para el contexto nacional, cuya incidencia en el comportamiento de algunos estudiantes es innegable, en tanto materializan en aulas de clase realidades concurrentes en sus familias $\mathrm{o}$ su entorno comunitario.

La convivencia escolar es un aspecto fundamental del proceso formativo, en tanto no solo enseña a convivir, sino permite descubrir habilidades, demostrar valores y generar espacios interactivos de

respeto, armonía y paz, esenciales para lograr los propósitos de aprendizaje. Sin embargo, en el marco de las interrelaciones propias del contexto escolar, se presentan manifestaciones de violencia, agresión e indisciplina que entorpecen dichos objetivos.

El conflicto escolar, como aspecto inherente al ser humano, también está presente en los procesos interactivos propios del proceso formativo $\mathrm{y}$ es resultante de actitudes personales asociadas al ego o la intolerancia, sumadas a situaciones originadas en la interacción social desde un origen basado primordialmente en relaciones de poder o de carácter ideológico.

Una conducta recurrente del conflicto escolar está relacionada con la agresividad, representada en comportamientos antisociales presentes en múltiples escenarios cotidianos y evidenciada en actos destructivos, agresiones personales de tipo física o verbal, directo o indirecto, activa o pasiva.

El análisis de resultados permitió evidenciar diversidad de conflictos, mayormente físicos y menormente verbales, así como manifestaciones de intolerancia, abuso de confianza, egoísmo e irrespeto, con repercusiones en su formación académica y personal. Además, la intervención docente en la mediación de los conflictos no es regular, ni obedece a criterios unificados por medio de estrategias debidamente estructuradas.

Por lo anterior, se hace necesario mejorar las relaciones, promover la convivencia de todos los miembros de la comunidad, generar cambios estructurales de carácter sociocultural, regular la resolución de conflictos a través de políticas educativas y mitigar situaciones con incidencia negativa en todo el estudiantado.

En este sentido, se requiere formular e implementar una propuesta de formación de mediadores escolares, como estrategia para buscar una reducción significativa de diferentes tipos de conflicto escolar índices, así como de su manifestación en agresiones, burlas o rupturas del tejido social.

Dicha estrategia debe estar soportada en la Ley 1620 del 2013, por medio de la cual se crea Sistema Nacional de Convivencia Escolar, como instrumento para incidir positivamente en aspectos relacionados con formación para ejercer adecuadamente toda serie de derechos humanos, educación para la sexualidad, prevención y mitigación de violencia escolar; así como para disminuir situaciones designadas como Tipo II: Agresión o acoso escolar (Decreto $N^{\circ} 1965$ de Sep. 2013) por parte del Ministerio de Educación 
Nacional de Colombia.

Finalmente, es importante establecer rasgos comunes en diferencias socioculturales generadoras de patrones conductuales negativos en adolescentes, a fin de destacar implicaciones arraigadas en diversos entornos socio educativos. De esta forma, es posible determinar otros tipos de variables de suma importancia en el proceso de implementación de planes, programas o proyectos de intervención socioeducativa, como estrategia de prevención de la conducta antisocial durante la época escolar.

\section{Reconocimiento}

Un reconocimiento muy especial a la Universidad de Pamplona (Colombia) y al grupo de Investigación Gestión Integral del Territorio por el apoyo al desarrollo de esta investigación.

\section{Referencias}

Abenza, A. (2015). La Violencia Escolar en los Centros de Educación Secundaria de la Región de Murcia: La Voz del Alumnado. Tesis. Universidad de Murcia, 1 - 334.

Aguilar, T., \& Ariza, J. (2015). La Resolución de Conflictos Escolares desde los Derechos Humanos: El Gran Viaje en el Aula. Tesis. Universidad Distrital Francisco José de Caldas, 1 - 77.

Andreu, J., Peña, M., \& Penado, M. (2013). Impulsividad cognitiva, conductual y no planificadora en adolescentes agresivos reactivos, proactivos y mixtos. Anales de Psicología. 29 (3), 734 - 740. DOI: http://dx.doi.org/10.6018/analesps.29.3.175691

Arredondo, A. (2015). Convivencia escolar: Una mirada desde la concepción humanista a la situación en Colombia. Clave Social, 4 (1), $\quad 50 \quad 61$. http://repository.lasallista.edu.co:8080/ojs/index.php/ENClave/article/view/951/898

Bahamón, C., Pacheco, M., \& Sáenz, J. (2015). Creencias y prácticas de la comunidad educativa de la IED Marruecos y Molinos, frente a la convivencia escolar. Tesis. Universidad de la Sabana, $1-68$.

Bustos, E. (2009). La conducta agresiva del niño en edad infantil. Revista digital innovación y experiencias educativas, 15(1), 1 9.https://archivos.csif.es/archivos/andalucia/ensenanza/revistas/csi csif/revista/pdf/Numero_15/EMILIA_BUSTOS_2.pdf

Caballero, M. (2010). Convivencia escolar. Un estudio sobre buenas prácticas. Revista de Paz y Conflictos, $\mathrm{N}^{\circ}$ 3, 154169.https://www.redalyc.org/articulo.oa?id=205016387011

Cabrales, L., Contreras, N., Gonzáles, L., \& Rodríguez, Y. (2017). Problemáticas de Convivencia Escolar en las Instituciones Educativas del Caribe colombiano: Análisis desde la Pedagogía Social para la Cultura de Paz. Tesis. Universidad del Norte, 1 145.
Cajiao, F. (2015). Gestión y Pedagogía. El Tiempo. https://www.eltiempo.com/archivo/documento/CMS15526842

Contini, E., Cohen, S., Coronel, C., \& Mejail, S. (2012). Agresividad y Retraimiento en Adolescentes. Ciencias Psicológicas, VI (1), p. 17 - 28.http://www.scielo.edu.uy/pdf/cp/v6n1/v6n1a03.pdf

De Souza, L. (2009). Una Mirada Genérica de los Conflictos. Universidad Autónoma de Barcelona. Contribuciones a las Ciencias Sociales. $1-9$.

www.eumed.net/rev/cccss/04/lsb.htm

Domínguez, R., y García, S. (2003). Introducción a la Teoría del Conflicto en las Organizaciones. Universidad Rey Juan Carlos, p. 1 -54 .

file:///E:/Downloads/INTRODUCCION_A_LA_TEORIA_DEL_ CONFLICTO_EN_LAS_ORGA\%20(3).pdf

García, A., y Ferreira, G. (2005). La convivencia escolar en las aulas. International Journal of Developmental and Educational Psychology, 2(1),163-183. https://www.redalyc.org/articulo.oa?id=3498/349832309012

González, P., y Rojas, D. (2014). Convivencia Escolar y Conflicto en el Aula: un Estudio Descriptivo sobre las Relaciones entre Jóvenes y Profesores de dos Liceos de Enseñanza Media. Tesis. Universidad de Chile, $1-188$.

González, R. (2017). Estrategias para Abordar los Conflictos en el Aula de Clase. Tesis. Universidad de Antioquia, Colombia, 1- 185.

López, C., Carvajal, C., Soto, M., y Urrea, P. (2013). Factores asociados a la convivencia escolar en adolescentes. Educ. Educ, 16 (3), 383

410.http://www.scielo.org.co/pdf/eded/v16n3/v16n3a01.pdf

Marshall, C., y Rossman, G. (1989). Designing qualitative research. 5th edición. Newbury. Park, CA: Sage, 1 - 321. DOI: 10.1057/omj.2011.23

Marrugo, G., Gutiérrez, J., Concepción, I., y Concepción, M. (2016). Estrategia de Convivencia Escolar Para la Formación de Jóvenes Mediadores de Conflictos. Escenarios, 14 (1), 72 - 84.DOI: https://doi.org/10.15665/esc.v14i1.879

Melo, F., Montaño, E., Mora, J., y Pavas, M. (2009). Dinámica del conflicto en la Institución Educativa Nuestra Señora del Carmen, Corregimiento El Carmen Municipio de San Lorenzo - Nariño. Tesis. Universidad de La Salle, Colombia, 1 - 198

Mendoza, B., Cervantes, A., y Pedroza, F. (2016). Acoso escolar: diferencias en contextos educativos rural y urbano, en alumnado adolescente. Investigación y Ciencia, 24 (67), 1 5.www.redalyc.org/articulo.oa?id $=67446178009$

Mina, L., y Rangel, C. (2012). El buen trato, estrategia para la sana convivencia entre la comunidad educativa IEPS. Informe de gestión, programa Trabajo Social. Universidad de Cartagena, 1 70.

Ortiz, A. (2014). Mediación Docente ante Situaciones de Conflicto en la I.E Mariscal Robledo. Educación y Pedagogía, 1 (5), 65 - 73. http://ayura.udea.edu.co:8080/jspui/bitstream/123456789/987/1/P B0831.pdf

Patiño, C. (2014). Apuntes para una historia de la educación en Colombia. Actual Pedagogía, 64 (1), 261 - 265. DOI: https://doi.org/10.19052/ap.3209 
Pérez, E., y Gutiérrez, D. (2016). El Conflicto en las Instituciones Escolares. Ra Ximhai, $12 \quad$ (3), 163 - 180. http://www.redalyc.org/articulo.oa?id=46146811010

Salazar, R. (2010). La Educación Rural un Reto Educativo. http://www.docentes.unal.edu.co/lgonzalezg/docs/LaEducacionR uralunRetoEducativo.pdf.

Samper, P., Tur, A., Mestre, V., y Cortés, M. (2008). Agresividad y afrontamiento en la adolescencia. Una perspectiva intercultural. International Journal of Psychology and Psychological Therapy, 8 (3), 431- 440. https://www.researchgate.net/publication/242645612

Sandoval, M. (2014). Convivencia y clima escolar: claves de la gestión del conocimiento. Última Década, (41), 153 -178. DOI: http://dx.doi.org/10.4067/S0718-22362014000200007

Silva, G. (2008). La Teoría del Conflicto: Un Marco Teórico Necesario. Prolegómenos. Derechos y Valores, XI (22), 29 - 43. http://www.redalyc.org/articulo,oa?id=87602203

Torregrosa, M., Inglés, C., y García-Fernández, J. (2011). El Comportamiento Agresivo Como Predictor del Autoconcepto: Estudio con una Muestra de Estudiantes Españoles de Educación Secundaria Obligatoria. Psychosocial Intervention, 20 (2), 201201.DOI: http://dx.doi.org/10.5093/in2011v20n2a8 